\title{
Cold Trapped Atoms: A Mesoscopic System*
}

\author{
Kerson Huang \\ Department of Physics and Center for Theoretical Physics \\ Massachusetts Institute of Technology \\ MIT-CTP\#3048
}

\begin{abstract}
The Bose-Einstein condensates recently created in trapped atomic gases are mesoscopic systems, in two senses: (a) Their size fall between macroscopic and microscopic systems; (b) They have a quantum phase that can be manipulated in experiments. We review the theoretical and experimental facts about trapped atomic gases, and give examples that emphasize their mesoscopic characters. One is the dynamics of collapse of a condensate with attractive interactions. The other is the creation of a 1D kink soliton that can be used as a mode-locked atom laser.
\end{abstract}

\section{Mesoscopic systems}

A mesoscopic system has two distinctive characteristics:

- It contains many more particles than a microscopic system, but is small enough for us to manipulate in the laboratory.

- It possesses a quantum phase that we can control.

The term "mesoscopic" has been associated with "quantum dots," in which the passage of electrons can be controlled singly. Now we have new

*Expanded version of an invited talk given at the third joint meeting of Chinese physicists worldwide, Chinese University of Hong Kong, August, 2000. 
mesoscopic systems, with the achievement of Bose-Einstein condensation in a system of trapped atoms. These Bose condensates typically contain the order of $10^{6}$ atoms, in a magnetic or optical trap, at a temperature of the order of $10^{-7} \mathrm{~K}$. After a brief summary of known facts, we shall give examples of both aspects mentioned above. For more details and literature, we refer to recent review papers on experiments [1] and theory [2].

\section{The Bose condensate}

As we know, there are two types of elementary particles: fermions of halfinteger spin, which cannot occupy the same state, and boson of integer spin, which love to be in the same state. Bound states of elementary particles, such as atoms and molecules, also fall into these categories. The bosonic atoms we are dealing with include ${ }^{87} \mathrm{Rb},{ }^{23} \mathrm{Na},{ }^{7} \mathrm{Li}$, and atomic hydrogen.

In the ground states all bosonic atoms occupy the same state; forming the Bose-Einstein condensate. What makes this an identifiable entity is its stability, arising from the quantum-mechanical fact that atoms are indistinguishable. In Fig. 1 we illustrate how this reduces the number of excited states. The situations illustrated would represent three different states, if the particles were distinguishable. But for indistinguishable particles they are one and same the same state. Because of this, there are fewer ways to excite the condensate, and hence it has a high degree of stability.

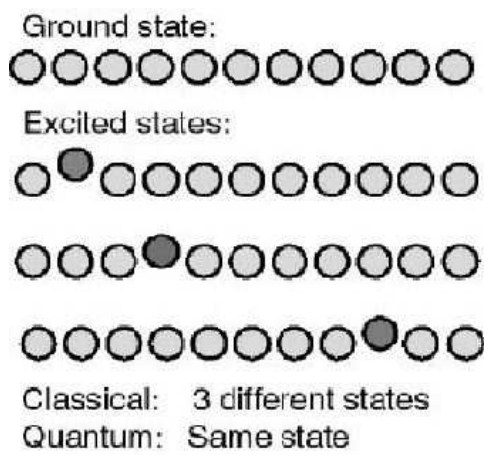

Figure 1: The indistinguishability of particles reduces the number of excited states, and makes the condensate stable.

In fact, when the temperature is raised from absolute zero, there remains a finite fraction of particles in the condensate. The condensate fraction de- 
creases with rising temperature, and disappears only above a finite temperature $T_{c}$, the critical temperature of the Bose-Einstein condensation.

In contrast, for "Boltzmann statistics", the ground state wave function is the same, but there will be a high density of excited states. In this case, no condensate exists at any finite temperature. That is, the transition temperature is at absolute zero.

The process of Bose-Einstein condensation is illustrated in Fig. 2.

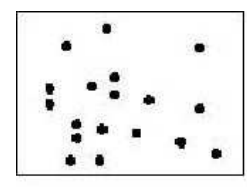

\section{High T}

Atom are like

billiard balls

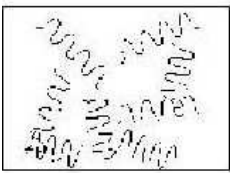

$T=T_{c}$

Wave functions overlap

Condensate begins

to form

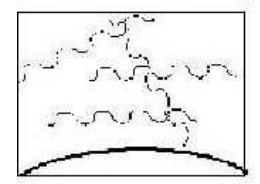

$T<T c$

Condensate in

equilibrium with

thermal cloud

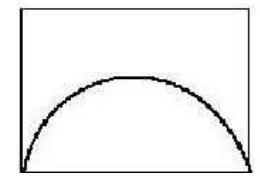

$T=0$

Pure condensate

System is one

giant matter wave

Figure 2: The approach to Bose-Einstein condensation.

\section{Experimental techniques}

The process to achieve BEC in the laboratory is illustrated in Fig. 3. An atomic beam was slowed down by laser cooling, and a magnetic trap was turned on. The spin up atoms are confined, while the spin down one are expelled. Final cooling is achieved through evaporation and rethermalization. To evaporate the fast atoms, rf light of appropriate frequency is turned on 


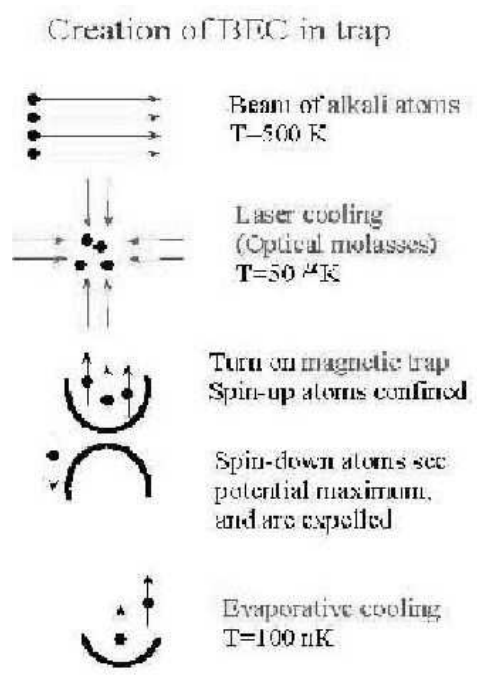

Figure 3: Experimental steps in the trapping and cooling of atoms.

to induce spin flip. In the final condensate, we have typically

$$
\begin{aligned}
N & \sim 10^{6} \\
V & \sim 10^{-6} \mathrm{~cm}^{3} \\
\frac{N}{V} & \sim 10^{12} \mathrm{~cm}^{-3}
\end{aligned}
$$

The density is some seven orders of magnitude smaller than that of air in the atmosphere.

A brief history of Bose-Einstein condensation in trapped gases is given Fig. 1.

\section{Helium clusters}

Long before trapped atoms, of course, Bose-Einstein condensation had been seen, though indirectly, in the superfluidity of liquid ${ }^{4} \mathrm{He}$. Andronikashvili performed the classic experiment on the superfluid fraction, by measuring the moment of inertia of a stack of rotating plates immersed in liquid helium, as a function of temperature. The idea was that the normal fluid would be dragged into rotation with the plates, but the superfluid component would not participate in the motion. 


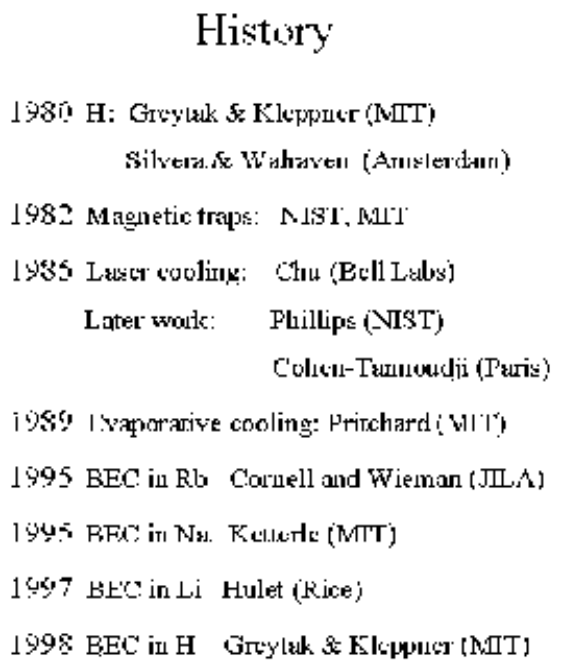

Figure 4: History of atom trapping and cooling.

The ultimate miniaturization of the Andronikashvili experiment has now been done [3] in ${ }^{4} \mathrm{He}$ clusters of a few hundred atoms, with the rotating plates replaced by a rotating atom of ${ }^{3} \mathrm{He}$. Superfluidity was reported in a cluster with 60 atoms. This is a very interesting mesoscopic system, but beyond the scope of our discussion here.

\section{Quantum coherence}

For an ideal Bose gas, the condensate is made up of particles in the same single-particle quantum state. When there are interparticle interactions, single-particle states are no longer meaningful; but we can define the condensate wave function as the quantum amplitude for removing a particle from the ground state:

$$
\psi(\mathbf{r}, t)=\sqrt{n_{s}} e^{i \phi}
$$

where $n_{s}$ is the condensate density, and $\phi$ is the quantum phase of the condensate.

The quantum coherence of the condensate is a remarkable thing. In experimental traps, the condensate can have a spatial extension as large as 1 $\mathrm{mm}$, which is almost visible to the naked eye, and yet this glob of matter is 
characterized by a single wave function, with a single phase. This has been demonstrated experimentally by switching off the magnetic trap, cutting the condensate in half with a laser "knife," and allowing the two halves to fall under gravity. The two condensates expand as they drop, and eventual overlap in space, creating a interference pattern, as illustrated in Fig. 5. An actual photograph of the interference fringes in shown in Fig. 6.

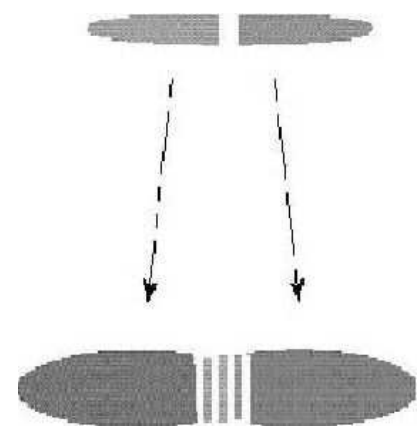

Figure 5: A condensate in free fall is cut by a laser knife, and the two halves continue to fall, expand, and overlap.

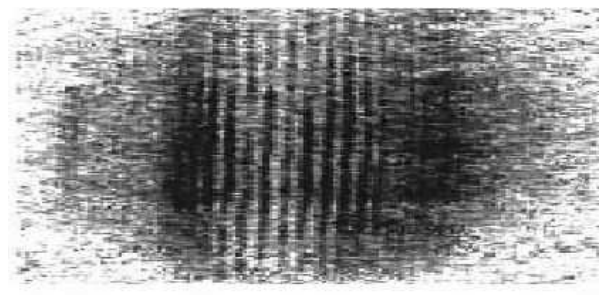

$$
\text { Fringe spacing }=\lambda / 2=15 \mu m
$$

Figure 6: Interference fringes in two overlapping condensates of sodium atoms. (Courtesy W. Ketterle.)

We can describe two non-overlapping free condensates by wave functions that are nearly plane waves:

$$
\begin{aligned}
& \psi_{1}=C_{1} e^{i\left(\mathbf{k}_{1} \cdot r-\omega_{1} t\right)} \\
& \psi_{2}=C_{2} e^{i\left(\mathbf{k}_{2} \cdot \mathbf{r}-\omega_{2} t\right)}
\end{aligned}
$$


When they overlap, they become one condensate with wave function

$$
\psi=\psi_{1}+\psi_{2}
$$

The density of the system is given by

$$
|\psi|^{2}=\left|C_{1}\right|^{2}+\left|C_{1}\right|^{2}-2 \operatorname{Re}\left[C_{1}^{*} C_{2} e^{\left.i\left(\mathbf{k}_{1}-\mathbf{k}_{2}\right) \cdot \mathbf{r}-i\left(\omega_{1}-\omega_{2}\right) t\right)}\right]
$$

where the last term exhibits the interference fringes. From Fig. 目, the fringe spacing gives a half wavelength $\lambda / 2=15 \mu \mathrm{m}$, which corresponds to a relative wave number

$$
k_{1}-k_{2}=\frac{2 \pi}{\lambda}
$$

The relative velocity of the two condensates was therefore given by

$$
v_{1}-v_{2}=\frac{\hbar}{m}\left(k_{1}-k_{2}\right)=0.05 \mathrm{~cm} / \mathrm{s}
$$

Atoms in the condensate in a magnetic trap can be ejected by inducing spin flips, through application of rf light. The expelled atoms fall under gravity as pulses of coherent matter, as shown in Fig. 7 . This is the first example of an atom laser [4.

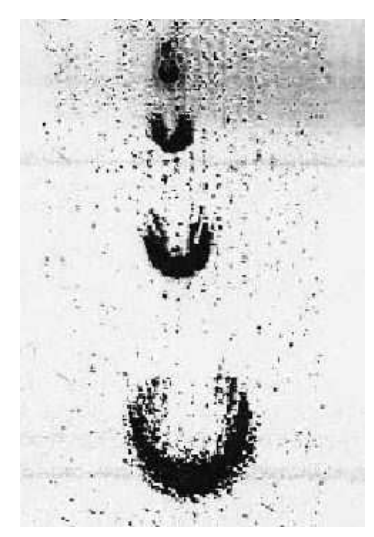

Figure 7: The first atom laser. (Courtesy W. Ketterle.) 


\section{Nonlinear Schrödinger equation}

The condensate wave function satisfies a nonlinear Schrödinger equation (NLSE), also called the Gross-Pitaveskii equation, in a mean-field approximation that neglects the coupling to the thermal cloud:

$$
i \hbar \frac{\partial \psi}{\partial t}=\left(-\frac{\hbar^{2}}{2 m} \nabla^{2}+V(r)+g|\psi|^{2}\right) \psi
$$

with

$$
g=\frac{4 \pi \hbar^{2} a}{m}
$$

where $a$ is the S-wave scattering length. The normalization condition

$$
\int d^{3} r|\psi|^{2}=N
$$

determines the number of particles in the condensate. This is a constant of the motion, but its value cannot be changed by convention, contrary to that for an ordinary wave function, because the equation is nonlinear.

\section{The scattering length}

The scattering length $a$ is illustrated in Fig. 8. The reduced relative wave function of two atoms, as a function of the separation $r$, approaches a linear asymptote beyond the range of the potential. The scattering length is the the intercept of the asymptote on the $r$ axis.

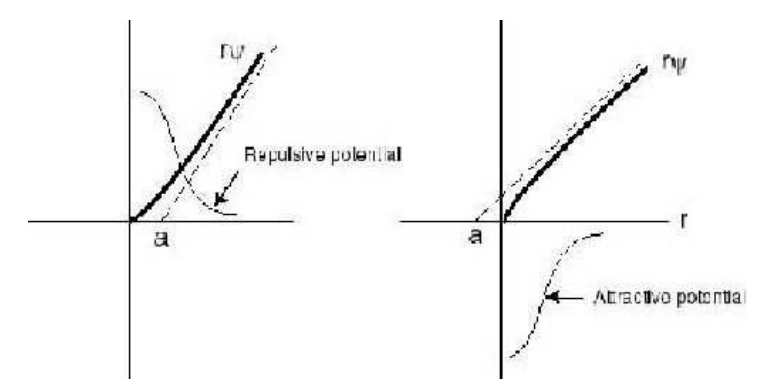

Figure 8: Scattering length a for potentials without bound states. 
When the potential has no bound state, as illustrated in Fig. 9, a repulsive potential give $a>0$, and an attractive potential give $a<0$. When there are bound states in a attractive potential, however, the scattering length can have either sign. As the potential gets more attractive, it changes sign whenever a bound state occurs.
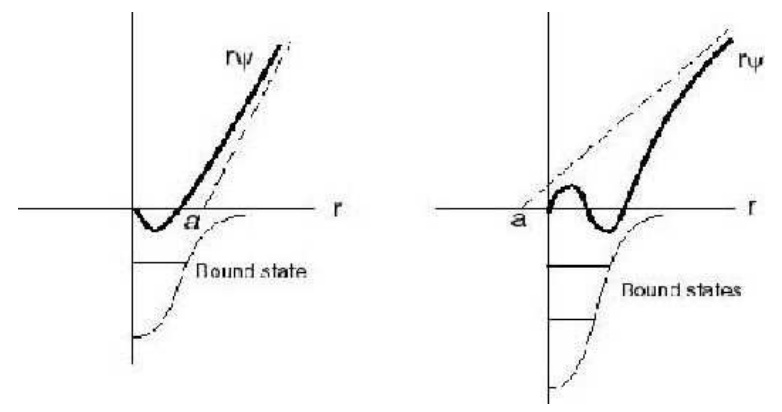

Figure 9: Scattering length a for potentials without bound states.

One of the exciting recent developments is that the scattering length can be tuned, from $-\infty$ to $\infty$, by changing an external magnetic field, through the Feshbach resonance in atomic scattering, which is similar to a bound state [5]. This realizes the usual thought experiment of changing the coupling constant in the Hamiltonian.

It should be emphasized that $a$ is a parameter that describes scattering in $3 \mathrm{D}$ in the limit of zero energy, where the shape of the potential is irrelevant. At higher energies, the shape of the potential does make a difference, and one must include other parameters, such as the effective range [6].

\section{Quantum tunneling}

The trapped atoms that form a Bose condensate at such low temperatures would have solidified in free space. In the trap, the zero-point motion kept them apart. In the case of ${ }^{6} \mathrm{Li}$, which has negative scattering length, the condensate is metastable, and decays via quantum tunneling, if the total number of atoms $N$ is sufficiently small. If $N$ exceeded a critical number, however, the system collapses [7, 8]. Let us discuss each of these scenarios. 
For negative scattering length, we have $g<0$. Let us denote

$$
G=-g=-\frac{4 \pi \hbar^{2} a}{m}
$$

We can see qualitatively that in free space the condensate collapses in spatial dimension $D>2$. The energy density of system is

$$
E=\frac{\hbar^{2}}{2 m}|\nabla \psi|^{2}-G|\psi|^{4}
$$

Suppose the system is confined to a region of dimension $R$. Then $\left.\psi\right|^{2} \sim$ $N / R^{D}$, so

$$
E \sim \frac{\hbar^{2}}{2 m} \frac{N}{R^{D+2}}-\frac{g N^{2}}{R^{2 D}}
$$

Thus $E \rightarrow-\infty$ when $R \rightarrow 0$, if $D>2$.

If there is a confining potential, then collapse happens only if $N$ is sufficiently large, because there is a potential barrier against collapse. To see this, consider the Hamiltonian corresponding to (8):

$$
H[\psi]=\int d^{3} r\left[-\frac{\hbar^{2}}{2 m} \psi^{*} \nabla^{2} \psi+V(r) \psi^{*} \psi-\frac{G}{2}\left(\psi^{*} \psi\right)^{2}\right]
$$

and choose a harmonic oscillator potential

$$
V(r)=\frac{1}{2} m \omega^{2} r^{2}
$$

For qualitative arguments, let us consider a confined wave function of a Gaussian shape

$$
\psi_{0}(r)=C_{0} \exp \left(-\alpha r^{2} / d_{0}^{2}\right)
$$

where

$$
d_{0}=\sqrt{\frac{\hbar}{m \omega}}
$$

The parameter $\alpha$ describes the width of the Gaussian. A Gaussian narrower than the harmonic oscillator wave function corresponds to $\alpha>1$. We can then replace $-\frac{\hbar^{2}}{2 m} \nabla^{2} \psi \rightarrow \chi(r) \psi_{0}$, where

$$
\chi(r)=\frac{\hbar^{2}}{2 m d_{0}^{2}}\left[3 \alpha-\left(\frac{r}{d_{0}}\right)^{2} \alpha^{2}\right]
$$


Thus the Hamiltonian can be represented in the form

$$
H[\psi]=\int d^{3} r \Omega_{r}(\psi)
$$

where the "field potential" $\Omega_{r}(\psi)$ is given by

$$
\Omega_{r}(\psi)=[V(r)+\chi(r)]|\psi|^{2}-\frac{G}{2}|\psi|^{4}
$$

This potential is plotted as a function of $|\psi|$ for different $r T$, in the right panel of Fig. 10. There is an energy barrier with height

$$
W_{r}=\frac{1}{G}[V(r)+\chi(r)]^{2}
$$

which is nonzero at $r=0$, because $\chi(0) \neq 0$. At large $|\psi|$ the field potential tends to $-\infty$. In reality, of course, the NLSE ceases to be valid somewhere along the drop, for other physical effects, such as solidification, come in.

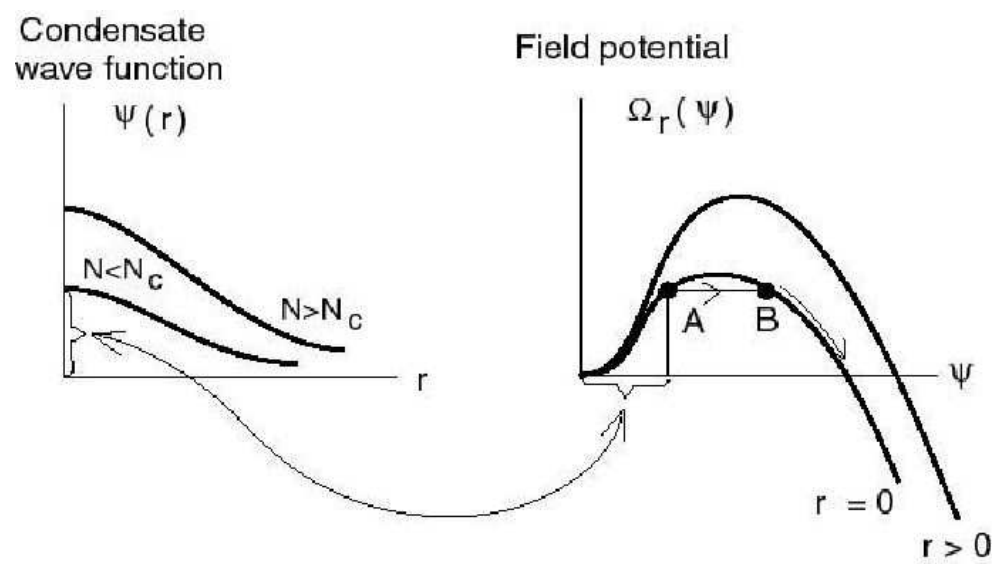

Figure 10: The field potential as seen by the condensate wave function. Local quantum tunneling $A \rightarrow B$ occurs across an energy barrier. If the wave function at $r$ goes over the energy barrier, then there is local collapse at that point.

A typical initial wave function $\psi_{0}(r)$ is shown on the left panel of Fig. 10. At a given $r$, we can measure the wave function on the left panel, and transfer it to the horizontal axis on the right panel. If it lands to the left of the barrier 
maximum for the particular $r$, then the system at that point is classically stable, but can decay via quantum tunneling as indicated by the classically forbidden path $A \rightarrow B$. If it lands to the right, the system at that $r$ will rapidly collapse to a state of high density.

Since $\psi_{0}(r)$ has a maximum at $r=0$ with value proportional to $\sqrt{N}$, the system will decays local tunneling, if it is classically stable at $r=0$. Otherwise, the system in a region about $r=0$ will rapidly collapse. The condition for stability against collapse is therefore $\Omega_{0}\left(\psi_{0}(0)\right)<W_{0}$. Within the Gaussian assumption, this corresponds to $N<N_{c}$, with

$$
N_{c}=\frac{3}{8} \sqrt{\frac{\pi}{\alpha}} \frac{d_{0}}{|a|}=\frac{0.665}{\sqrt{\alpha}} \frac{d_{0}}{|a|}
$$

In the experiments with ${ }^{7} \mathrm{Li}$ at Rice University [9], the parameters are

$$
\begin{aligned}
a & =-1.45 \mathrm{~nm} \\
d_{0} & =3 \mathrm{~m} \\
\frac{2 \pi}{\omega} & =6000 \mathrm{~s}
\end{aligned}
$$

With the choice $\alpha=1.8$ (See later), we obtain $N_{c} \approx 1200$ from (22).

For $N<N_{c}$, the condensate decays slowly via quantum tunneling, as indicate by the path $A \rightarrow B$ in Fig. 10. Since the tunneling path depends on $r$, the tunneling is a local phenomenon, proceeding at different rates at different distances from the center of the trap.

It should be noted that quantum tunneling is a phenomenon not included in the NLSE, which is a classical approximation. The tunneling probability can be calculated via a Feynman path integral, and in a WKB-type approximation can be obtained by solving the NLSE in imaginary time [7].

Qualitatively, we can describe the phenomenon as follows. The condensate locally makes a quantum transition from a low-density state $A$ to a high-density state $B$. The time at which this happens is random, and follows a statistical distribution. Since the total number of particles is conserved, what we see is that high-density droplets would "ooze out" of the condensate, as illustrated in Fig. 11. Once form, the droplets will tend to contract to states of even higher density, and will leave the trap through effects not included in our Hamiltonian, such as spin-flip scattering.

A more rigorous treatment of the field potential, independent of the Gaussian assumption, may be modeled after that of the "effective potential" in quantum field theory [10]. 


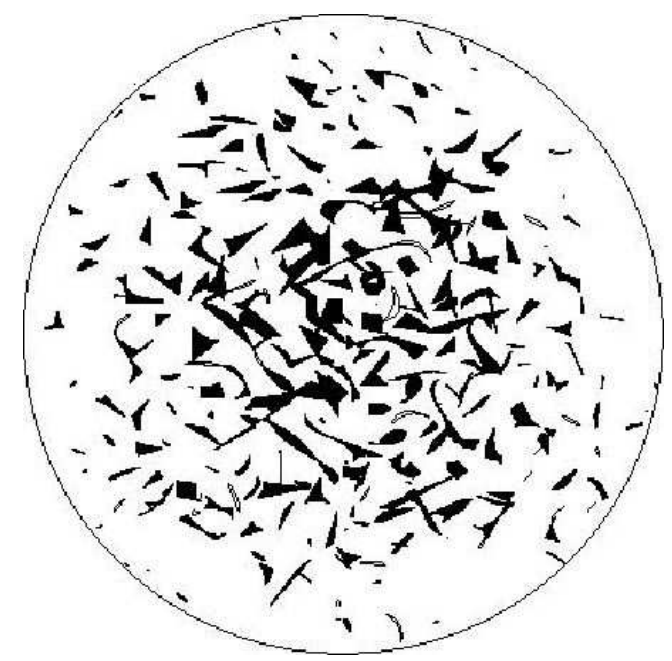

Figure 11: High-density droplets "ooze out" of the condensate via local quantum tunneling.

It should be noted that, unless $N \approx N_{c}$, the tunneling probability is very small. In reality, it would be masked by the decay of the condensate through spin-flip scattering, an effect that we have not taken into account.

\section{Collapse of the condensate}

When $\dot{N}>N_{c}$, the condensate with negative scattering length collapses. As we can see from Fig. 10, the collapse does not happen all at once over the entire condensate, but begins at the center of the condensate, where the density is highest. A black hole opens up at the center, in which, theoretically according to the NLSE, the density fluctuations becomes infinite, and the pressure becomes negative [7].

Numerical solutions of the NLSE have been obtained [8], with the perimeters (23). After the condensate was created in some arbitrary state, the wave function quickly adjusted itself to a Gaussian-like quasi-stationary state, with a width corresponding to $\alpha=1.8$. This situation persists infinitely if $N$ is sufficiently small.

If $N$ is larger than a critical value, then the quasi-stationary states lasts for a time, but then suddenly a finite volume about the center implodes, creating a black hole. Thereafter, waves of implosion arrives from all over 


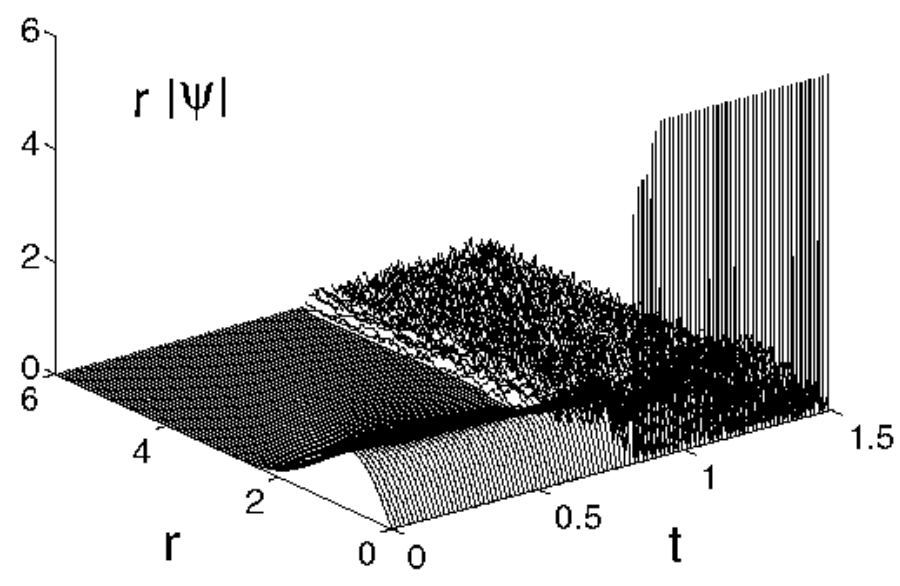

Figure 12: The condensate is stationary for a time, and suddenly collapses locally, near the center of the trap, opening up a black hole. The density in the black hole shoots up as time goes on, fed by waves of implosion, which make the density look turbulent. The density height is truncated because of finite grid size in the numerical simulation.

the condensate, whose density now appears to be turbulent. These features are summarized in Fig. 12, where $r|\psi|$ is plotted as a function of $r$ in unit of $d_{0}$, and $t$ in unit of $2 / \omega$, for a fixed $N$. By decreasing $N$ until the time of collapse goes to infinity, it was found that $N_{c} \approx 1200$.

In the experimental situation, the condensate in the trap can exchange atoms with an uncondensed thermal cloud, which contains far more atoms than the condensate. Equilibrium is reached when the chemical potentials equalize. The atoms can be depleted through two and three-body spin-flip collisions, and the latter becomes especially important where the density becomes large. These gain and loss mechanisms can be simulated by adding extra pure-imaginary terms to the NLSE, with a linear gain term, and a loss term proportional to $|\psi|^{4} \psi$. With these additions, the system settles down to steady state in which $N$ oscillates in growth-collapse cycles, as shown in Fig. 13. Again, we obtain $N_{c} \approx 1200$. 


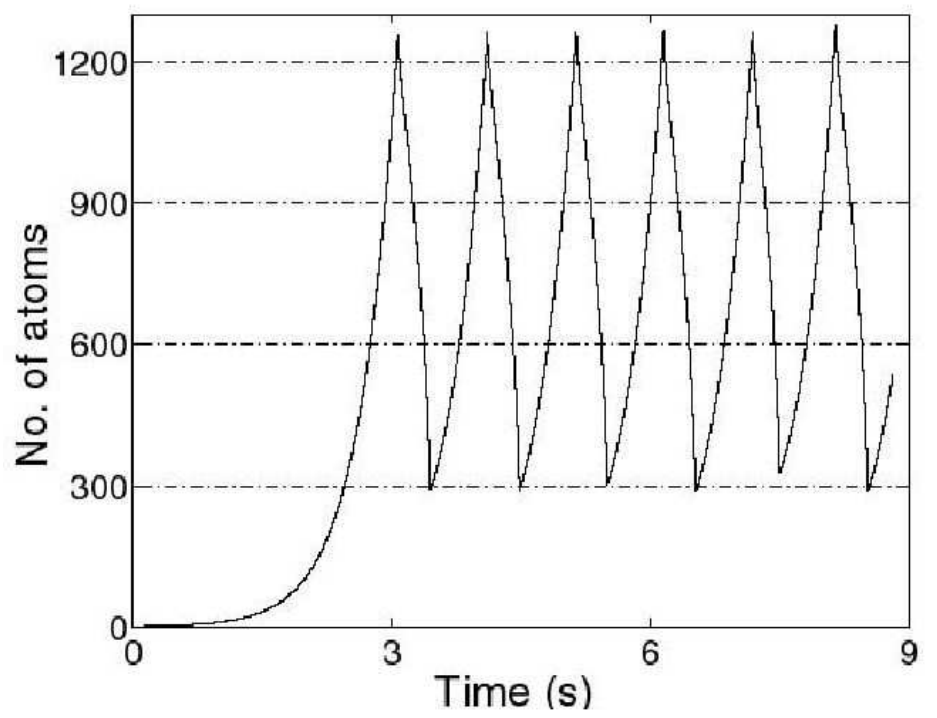

Figure 13: The condensate goes through steady-state growth-collapse cycles, in the presence of gain-loss mechanisms.

\section{Solitons in a condensate ring}

A spatial variation of the phase $\phi$ of the condensate wave function gives rise to a superfluid velocity

$$
\mathbf{v}_{s}=\frac{\hbar}{m} \nabla \phi
$$

and a supercurrent density

$$
j_{s}=n_{s} v_{s}
$$

Thus, flow patterns in the condensate can be created by "phase engineering." This has been done to create vortices [11, 12] and solitons [13.

One-dimensional solitons have been used in optical fibers for signal transmission over thousands of kilometers, with minimal loss. The electromagnetic field in an optical fiber obeys a NLSE of the same form as (8) [14]. Thus, it is natural to wonder whether we can create solitons in a $1 \mathrm{D}$ condensate. As illustrated in Fig. 14, the nonlinear term $g|\psi|^{2}$ represents an effective potential, which is attractive for $g<0$, and repulsive for $g>0$. $g<0$ As we 
saw earlier, the case does not lead to collapse in 1D. In this case, a localized wave packet created a attractive well of the same shape, hence it would trap itself into a bright soliton. In the case $g>0$, on the other hand, a "kink" configuration would self-trap, giving rise to a dark soliton.

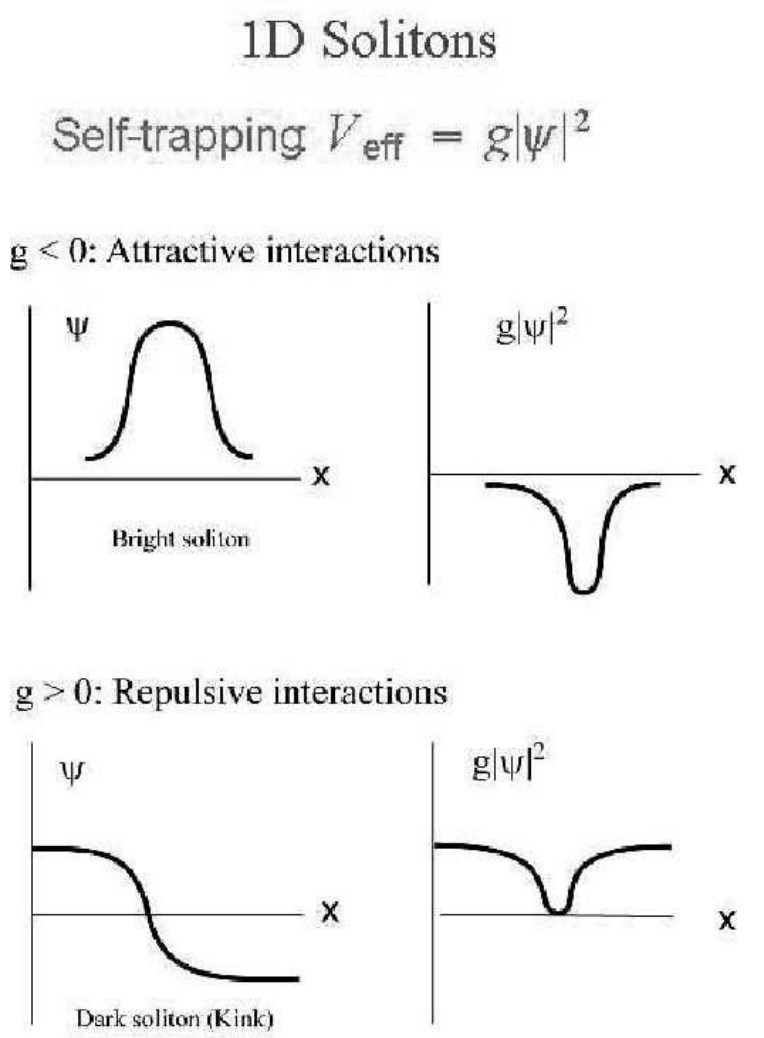

Figure 14: Formation of 1D solitons via "self-trapping".

Consider now a ring-shaped condensate. If the radius of the ring is much larger than the radius of the cross section, then we can regard the system as one-dimensional, in the sense that transverse excitations have a much high energy than the logitudinal ones, and may be ignored at low temperatures. However, the cross sectional radius may still be large compared to the scattering length, so that the system is still $3 \mathrm{D}$ with respect to interatomic scattering.

We concentrate on the repulsive case with $g>0$. The solution to the 
NLSE must satisfy the boundary condition

$$
\psi(\theta+2 \pi, t)=\psi(\theta, t)
$$

where $\theta$ is the angle around the ring. Let us write

$$
\psi(\theta, t)=f(\theta, t) e^{i \phi(\theta, t)}
$$

For a kink soliton, $f$ must have a zero, and so changes sign when $\theta$ increases by $2 \pi$. To satisfy the boundary condition, the phase must change by an odd multiple of $\pi$ in one complete revolution. The simplest case corresponds to a change of $\pi$ in the phase. This means that the lowest kink soliton has angular momentum per particle $\hbar / 2$, for the same mathematical reason that an electron has spin $1 / 2$.

The solution can be obtained analytically [16. The phase changes most rapidly at the location of the kink, and its slope there gives the kink a nonzero propagation velocity. Thus, the soliton is manifested as a dark spot moving around the ring with a characteristic velocity. The width of the soliton turns out to be proportional to $N^{-1 / 2}$, where $N$ is the number of atoms in the condensate.

\section{A mode-locked atom laser}

The first atom laser illustrated in Fig. 7 consists of pulses of coherent atoms. However, the different pulses do not have definite phase with respect to each other. There is an advantage to have a mode-locked laser — one in which the output pulses are phase coherent. In more recent experiments, a steady stream of output pulses has been achieved [15, but it is not clear whether the pulses are coherent with respect to each other.

We can use of our kink soliton to make a mode-locked atom laser 16. The physics is illustrated in Fig. 15. The ring of condensate is surround by a thermal cloud, which tends to maintain an equilibrium number of particles $N$ in the ring. Now suppose we use some stroboscopic device to take particles out the soliton, by enlarging its width, which is proportional to $N^{-1 / 2}$. Then particles will flow from the thermal cloud to the condensate to restore $N$, and thus restoring the width.

An important question is how to create the kink soliton, and whether it is robust against perturbations. In a simulation, the soliton was created by 

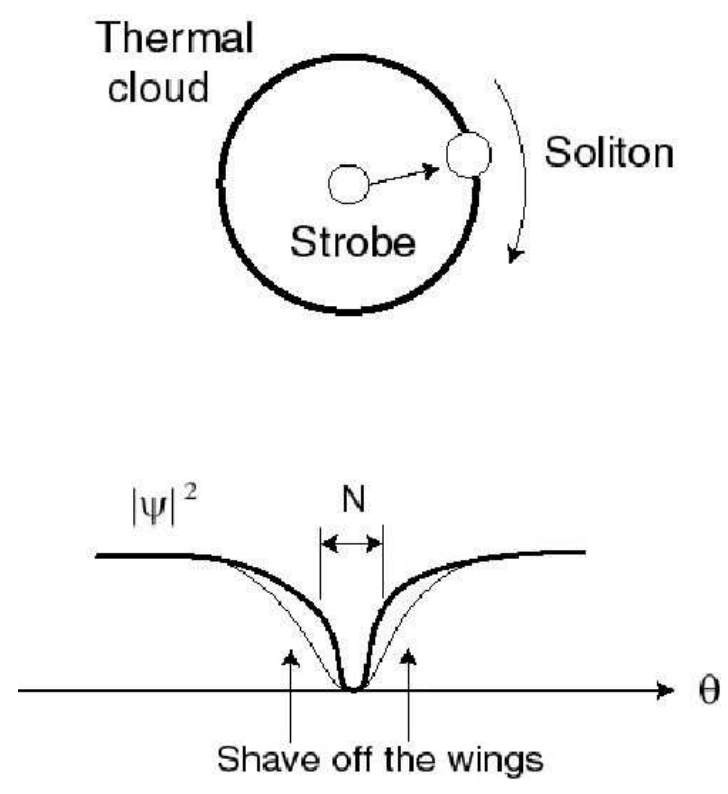

Figure 15: Mode-locked atom laser created by stroboscopically shaving off atoms at the kink of the dark soliton. The width grows back due to gain from the thermal cloud.

applying a moving momentary repulsive potential, such as that created by a laser beam, to create a hole in the condensate that moves at the characteristic soliton speed. This hole develops into the kink soliton, plus all kinds of excitations such as sound waves. To clean up the configuration, a periodic loss mechanism was introduced, in the form of am imaginary stroboscopic potential that also acts as outcoupler. At the same time, a gain mechanism was put in to simulate the thermal cloud. All unwanted excitations rapidly disappeared, leaving the kink soliton in steady-state equilibrium. The stability of the soliton come from topology - the fact that it has half-integer angular momentum per particle.

The creation and stabilization of the soliton is depicted in Fig. 16, where $|\psi|$ is plotted as a function of $\theta$ and $t$, with a contour plot projected onto the $\theta-t$ plane. The fact that we have in fact a kink soliton is indicted in Fig. 17, where the angular momentum per particle is plotted as a function of time. Finally, Fig. 18 shows the oscillation of the number of particles in the ring. The particles lost from the ring form the pulses in the mode-locked laser. They are coherent because the stroboscopic action is coherent. 


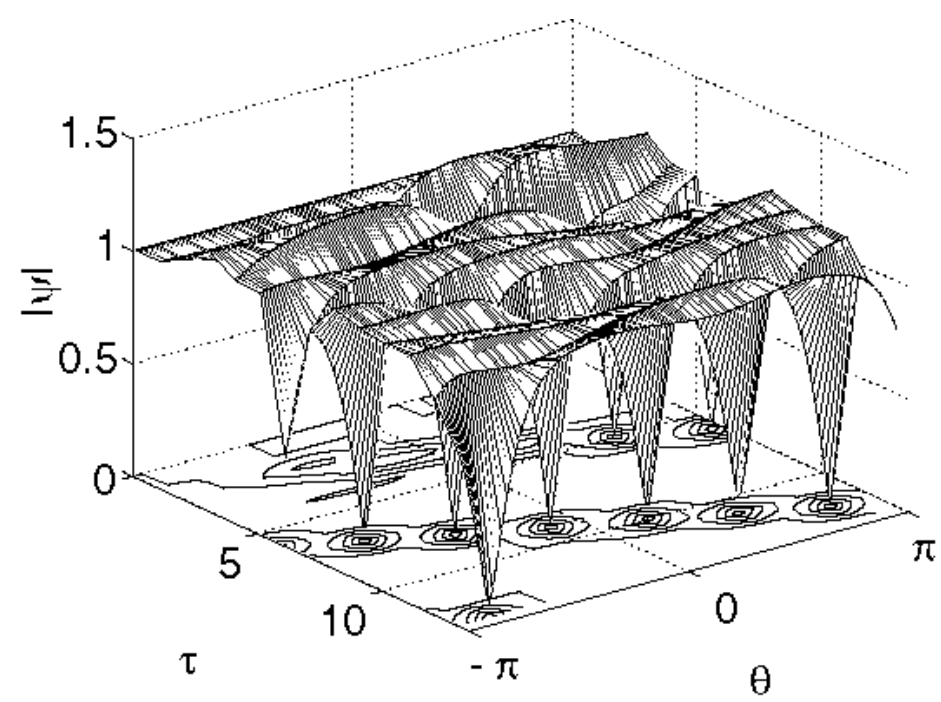

Figure 16: The kink soliton was created by stirring the condensate at its characteristic velocity, Other excitations also created in the stirring were cleaned up by a stroboscopic loss mechanism, which also serves as output coupler for the atom laser. The kink soliton survives because of topological stability.

\section{Acknowledgment}

This work is support in part by a U.S. Department of Energy cooperative agreement DE-FC02-94ER40818.

\section{References}

[1] W. Ketterle, D.S. Durfee, and D.M. Stumper-Kurn, in Proceedings.of Internaltional School of Physics, Enrico Fermi Course CXL, M. Ignaxcio, S. Strinagari, and C.E. Wieman, Eds. (Soc. Italiana di Fisica, Bologna, Italy, 1999).

[2] F. Dalfovo, S. Giorgini, L.P. Pitaevskii, and S. Stringari, Rev. Mod. Phys. 71, 463 (1999).

[3] S. Grebenev, J.P. Toennies, and A. F. Vilesov, Science, 279, 2083 (1998).

[4] M.-O. Mewes et. al., Phys. Rev. Lett. 78, 582 (1977). 


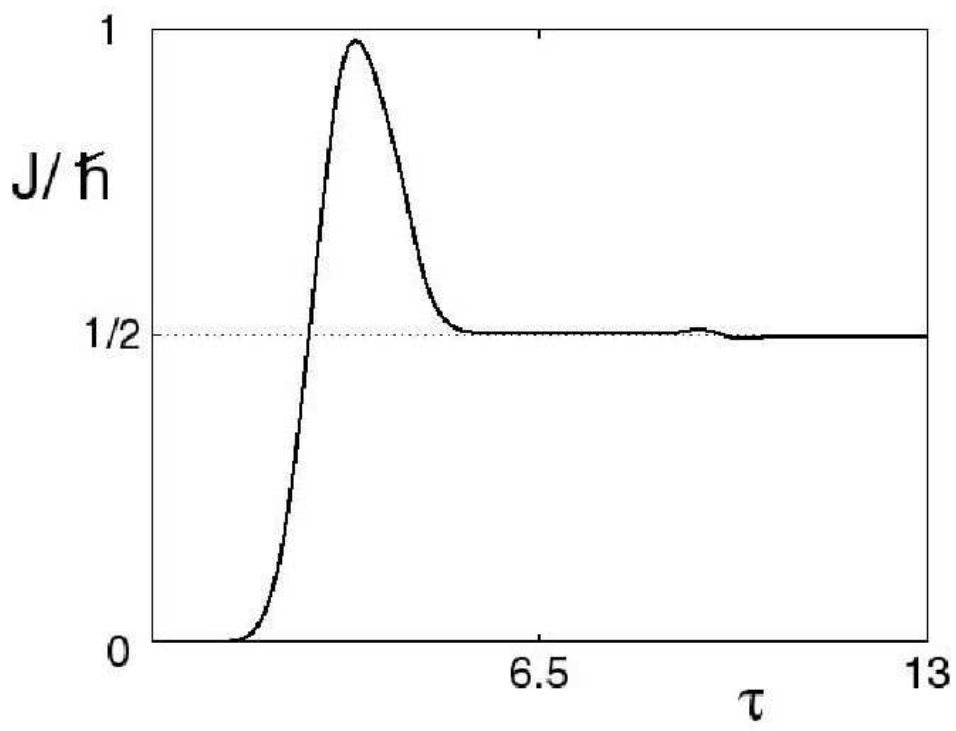

Figure 17: The angular momentum per particle as function of time. After transients die out, it approaches the half-integer value of a kink soliton.

[5] S. Inouye et. al., Nature, 392, 151 (1998);

[6] See K. Huang, Statistical Mechanics, 2nd ed. (Wiley, New York, 1987)., Sec.10.5.

[7] M. Ueda and K. Huang, Phys. Rev. A 60, 3317 (1999).

[8] A. Eleftheriou and K. Huang, Phys. Rev. A 61, 043601 (2000)..

[9] C. C. Bradley, C. A. Sackett, and R. G. Hulet, Phys. Rev. Lett. 78, 985 (1997); C. A. Sackett, H. T. C. Stoof, and R. G. Hulet, Phys. Rev. Lett. 80, 2031 (1998).

[10] See K. Huang, Quantum Field Theory, (Wiley, New York, 1998), Sec.15.7.

[11] J.E. Williams and M.J. Holland, Nature, 401, 568 (1999).

[12] M.R. Mathews et. al., Phys. Rev. Lett. 83, 2498 (1999).

[13] J. Denschlag et. al.. Science, 287, 97 (2000). 


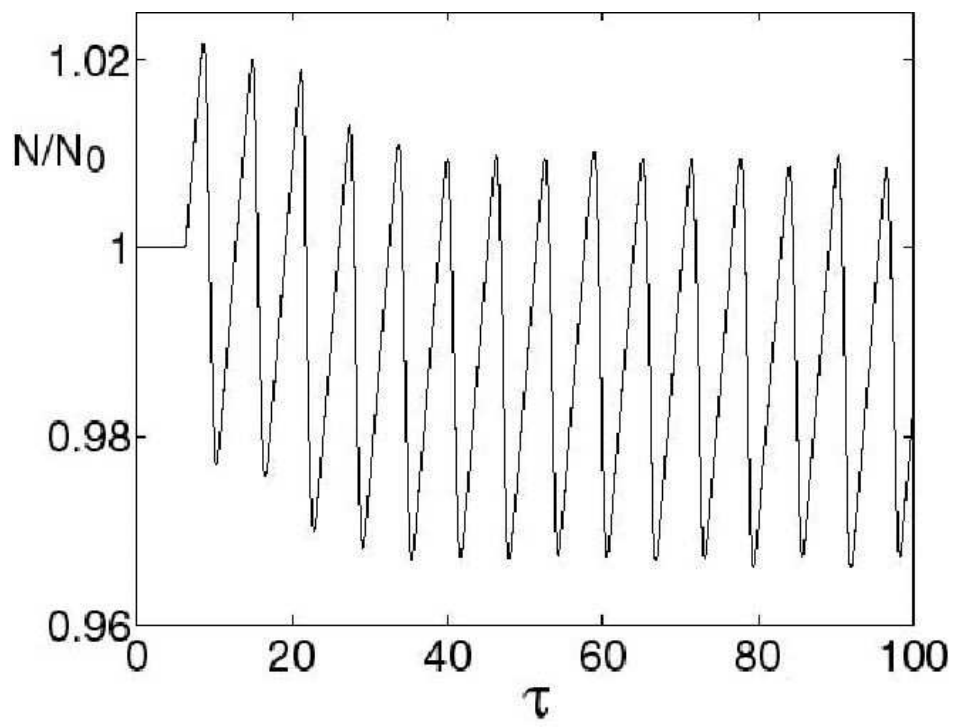

Figure 18: Number of atoms in the ring condensate exhibits steady-state coherent oscillations.

[14] A. Hasegawa, Optical Solitons in Fibers, 2nd ed. (Springer-Verlag, Berlin, 1995).

[15] B.P. Anderson and M.A. Kasevich, Science,27, 1686 (1998); I Bloch, T.W. Haensch, and T. Esslinger, Phys. Rev. Lett. 82, 3008 (1999); M Kozuma et. al. Sience, 286, 2309 (1999).

[16] P.D. Drummond, A. Eleftheriou, K. Huang, and K.V. Kheruntsyan, cond-mat/0002389. 